\title{
Cáncer serosopélvico de alto grado: evidencia sobre su origen en la trompa de Falopio
}

\author{
Felipe Serman V. ${ }^{1}$, Miguel Saavedra C. ${ }^{1}$ \\ ${ }^{1}$ Unidad de Oncología Ginecológica, Servicio de Ginecología y Obstetricia, Hospital de Carabineros. Santiago, Chile.
}

\section{RESUMEN}

Cáncer epitelial de ovario es una enfermedad altamente letal. Constituye la quinta causa de muerte por cáncer en mujeres a nivel mundial. El subtipo histológico más frecuente es el carcinoma seroso de alto grado. Este es el responsable de la alta letalidad de la enfermedad. Se presenta evidencia que respalda el origen tubario de este tipo histológico desde lesiones precursoras. A partir de estos datos se ha establecido que el cáncer tradicionalmente conocido como cáncer ovárico seroso de alto grado, el cáncer de trompa de Falopio y el carcinoma peritoneal primario, corresponden a una misma entidad nosológica: cáncer seroso pélvico de alto grado. Se revisa además la evidencia disponible para establecer que la salpingectomía podría constituir una medida de prevención para este tipo de cáncer.

\section{PALABRAS CLAVE: Cáncer seroso pélvico de alto grado, lesiones precursoras, trompa de Falopio, salpingectomía}

\section{SUMMARY}

Epithelial ovarian cancer is a highly lethal disease. It is the 5th cause of cancer death in women worldwide. The most common histologic subtype is the high-grade serous carcinoma. This is the responsible for the high lethality of the disease. Evidence supporting the tubal origin of this histological type from precursor lesions is presented. From these data it has been established that cancer traditionally known as serous high-grade ovarian cancer, cancer of the fallopian tube and primary peritoneal carcinoma, correspond to a single disease entity: pelvic serous high-grade cancer. We also check the available evidence to establish that the salpingectomy could be a preventive measure for this type of cancer.

KEY WORDS: Pelvic serous high-grade cancer, precursor lesions, fallopian tube, salpingectomy

\section{INTRODUCCIÓN}

El carcinoma ovárico es una enfermedad altamente letal (1). Más del $60 \%$ de las mujeres afectadas mueren a causa de esta enfermedad. En dos de cada tres casos está diseminada más allá de los órganos reproductivos en el momento del diagnóstico (2). En el mundo es la quinta causa de muerte por cáncer en mujeres. Anualmente, con una in- cidencia de 16 por 100 mil mujeres (3) y 225.000 nuevos casos, mueren 140.000 mujeres por esta causa $(3,4)$. En Chile, ha aumentado su tasa de incidencia (5,9 por 100.000 mujeres), y de mortalidad (4,4 por 100.000 mujeres) anuales (5).

El subtipo histológico más frecuente y letal es el carcinoma seroso de alto grado (CSAG) $(70 \%$ de todos los carcinomas ováricos, responsable del $90 \%$ de las muertes por cáncer ovárico) $(6,7,8)$. 
La mayoría de éstos son diagnosticados en etapa avanzada, con tasa de sobrevida a 5 años entre $9-34 \%$ (8). De alto grado indica su alta atipia nuclear y alto índice mitótico $(9,10)$. Se diferencia de otros subtipos por su naturaleza agresiva y albergar alteraciones genéticas únicas.

El cáncer ovárico epitelial (COE) se divide en las categorías Tipo I y Tipo II $(8,11,12,13,14)$ :

Tipo I: Tumores de bajo grado, confinados al ovario por períodos prolongados, clínicamente indolentes y genéticamente estables. Surgen de lesiones precursoras reconocibles (endometriosis o tumores borderline), y albergan las mutaciones KRAS, BRAF, CTNNB1, PTEN o ARID1A. Incluye carcinomas endometrioides, de células claras, mucinosos, serosos de bajo grado, y tumores borderline (serosos, mucinosos, endometrioides). Corresponde al $25 \%$ de todos los cánceres ováricos.

Tipo II: Neoplasias de crecimiento rápido, altamente agresivas. La mayoría son CSAG. Tienen alto nivel de inestabilidad genética (15), mutación del gen TP53 $(9,16)$ y de BRCA $(17,18)$. Son biológicamente agresivos desde su inicio, con propensión a metastizar desde lesiones primarias de pequeño volumen. Característicamente al momento del diagnóstico ya existe diseminación peritoneal extra anexial (11). Se pueden presentar clínicamente con compromiso predominante de ovario, trompa de Falopio o peritoneo, por lo que su denominación actual recomendada es carcinoma seroso pélvico de alto grado (CSPAG) $(16,19)$. Dada su precoz diseminación regional, reviste importancia la comprensión de sus fases iniciales, incluyendo condiciones precursoras (20).

\section{CARCINOGÉNESIS PÉLVICA SEROSA}

En mujeres con mutaciones BRCA (BRCA+) se realiza salpingo-ooforectomía bilateral reductora de riesgo (SORR) de cáncer ovárico $(21,22,23)$ con examen patológico ovárico y no exhaustivo de trompas de Falopio, buscando cáncer oculto.

En 12 pacientes BRCA+ sometidas a SORR, la sección y examen exhaustivo de las trompas de Falopio encontró $50 \%$ de displasia epitelial, versus ninguna lesión hiperplásica, displásica o neoplásica en las trompas de mujeres sin mutación. Las regiones displásticas presentaban fenotipo secretor, con pérdida de células ciliadas y adquisición de capacidad proliferativa (indicada por inmunoreactividad a Ki67) (24).

En 13 mujeres BRCA+ sometidas a SORR, el examen de las trompas empleando un protocolo específico de Sección y Examen Extensivo de la FIMbria (SEE-FIM), demostró un 38\% de carcinomas intraepiteliales serosos, y ninguno en los ovarios. El $80 \%$ de éstos se localizaban exclusivamente en la fimbria (25). Otros estudios con examen exhaustivo de las trompas demuestran la existencia de cánceres ocultos en la trompa de Falopio, la mayoría en la fimbria, en el 57 al $100 \%$ de las SORR en la población de mujeres BRCA+ $(26,27,28,29,30)$.

En 55 pacientes consecutivas con carcinoma pélvico seroso (ovárico, tubárico y peritoneal), no seleccionadas de acuerdo al status BRCA, el examen microscópico de las trompas de Falopio demostró carcinoma intraepitelial tubario (CIT) en 41 casos (75\%). El $93 \%$ se localizaban predominantemente en las fimbrias. En 5 de 5 CIT y carcinoma "ovárico" concurrente contenían idénticas mutaciones p53 (31). CIT coexistió con todas las formas de carcinoma seroso pélvico y se planteó como el origen de la mayoría de estos tumores $(31,32)$.

\section{VÍA DE LA TROMPA DE FALOPIO PARA LA PA- TOGÉNESIS DE LOS CÁNCERES SEROSOS DE ALTO GRADO}

STIC (CIT seroso por sus siglas en inglés) es un cáncer intraepitelial con potencial para diseminación, altamente proliferativo. Precede directamente al carcinoma seroso invasor. Histológicamente está compuesto de células secretoras con atipia significativa. Contiene mutaciones p53, con acumulación nuclear de la proteína p53 mutada en cerca del $80 \%$ de los casos, detectable con inmunohistoquímica $(33,34)$.

STIC tendría la capacidad de diseminarse más allá del sitio de origen (fimbria) con mínima invasión local, propiedad exclusiva de los carcinomas serosos iniciales. La proximidad de la fimbria a la superficie ovárica y cavidad peritoneal facilitaría la diseminación de clones tumorales más agresivos, que crecerían a mayor velocidad en esos sitios remotos (31).

En 45 pacientes consecutivas con carcinoma seroso peritoneal primario (CSPP) con examen de trompas con protocolo SEE-FIM se encontró compromiso del endosálpinx en $75 \%$, con STIC en $47 \%$. El análisis para mutaciones p53 demostró idéntica mutación p53 en STIC y en el tumor peritoneal (35). La revisión por estos autores de los casos de STIC reportados en la literatura, demostró ausencia de enfermedad en el seguimiento. Series grandes de SORR, muchas de ellas sin examen completo de la trompa de Falopio, con probablemente muchos STIC no diagnosticados, muestran baja tasa de evolución adversa, o sea, la mayoría de los STIC, si no todos, no alcanzan a diseminarse antes de ser extirpados. Se concluyó que la fimbria es la fuente de casi la mitad de los CSPP, y que el riesgo bajo de recurrencia de STIC lo identifica como un posible blanco para la detección y prevención del CSPAG (35).

En 87 casos consecutivos de cáncer pélvico seroso se analizaron las trompas con protocolo SEE-FIM y se determinó el inmunofenotipo de las 
lesiones (36). Se usó PAX8 (marcador del sistema mülleriano (37) y de células secretoras de la trompa de Falopio (38), p73 (marcador del núcleo de las células ciliadas tubarias (39), y anticuerpos marcados dirigidos contra p53 y p16 (ambas proteínas con sobreexpresión en carcinomas serosos de alto grado $(40,41,42)$. STIC fueron difusamente inmunopositivos para PAX8 y p53, con positividad parcial para p16. STIC no presentó células p73positivas. Hubo localización nuclear intensa de PAX8 en 85 de 87 carcinomas serosos y p73 fue negativo en 75 de 87 casos. Hubo marcada tinción nuclear para p53 y tinción citoplasmática para p16 en 59 y 60 de 87 carcinomas serosos, respectivamente, concluyendo que en CSPAG, las lesiones peritoneales extraováricas, las lesiones ováricas, y las fímbricas, incluidos los STIC, comparten similar inmunofenotipo, lo que sugirió la existencia de una célula originaria común para todas las categorías, independiente del sitio de las lesiones (36).

CÉLULA SECRETORA DEL EPITELIO DE LA TROMPA DE FALOPIO, ORIGINARIA DE LOS CSPAG. "P53 SIGNATURE": EL PRECURSOR NO MALIGNO DEL STIC

En el epitelio fímbrico existen segmentos compuestos sólo por células de tipo secretor, no proliferativas e histológicamente benignas, con fuerte inmunoreactividad a p53 $(24,25)$, se les denominó "p53 signatures".

Son igualmente frecuentes en mujeres BRCA+ y en controles (sometidas a salpingo-histerectomía por causa uterina benigna), presentándose en aproximadamente un tercio en cada grupo, lo que sugiere que son un fenómeno "normal" (39). Además presentan fuerte tinción con el marcador de daño de DNA gama-H2AX (g-H2AX). Esta es una forma fosforilada de la histona H2AX, fosforilación que ocurre rápidamente en sitios de rotura de la doble hebra de DNA (43). La marcada tinción con g-H2AX es un hallazgo común a otros precursores epiteliales $(44,45)$. La presencia de "p53 signatures" en las fimbrias de mujeres normales demuestra que, bajo condiciones fisiológicas normales, las células epiteliales fímbricas experimentan daño genotóxico y gatillan una respuesta al daño de DNA; "p53 signatures" son más frecuentes en trompas con STIC, se han detectado en continuidad con éste y comparten la mutación p53. STIC puede distinguirse de "p53 signatures" por su capacidad proliferativa aumentada (demostrada por positividad de MIB1 y expresión de Cyclina E aumentada). Estas observaciones permitieron plantear que "p53 signatures" representan el precursor del CSPAG (39). La patogénesis involucraría daño del DNA, mutación p53, y pérdida progresiva del control del ciclo celular (39). p53 mutado se acumula en las células secretoras del epitelio $(46,47)$ y originaría los "p53 signatures". Con estos hallazgos se considera a la célula secretora del ETF (CSETF) como la probable célula de origen del CSPAG.

\section{MODELO DE CARCINOGÉNESIS PÉLVICA SE- ROSA}

En el epitelio fímbrico, la combinación de mutaciones TP53 y estrés genotóxico lleva a expansión clonal de las células secretoras, formando una lesión precursora preneoplásica o "p53 signature". Golpes genéticos adicionales en la ausencia de un TP53 funcional hacen que estas células adquieran capacidad proliferativa, facilitando su progresión a STIC. Este tiene la capacidad de diseminarse desde la fimbria a estructuras pélvicas adyacentes (superficie ovárica, serosa uterina, membranas peritoneales, cavidad peritoneal (39) (Figura 1). El carcinoma invasor constituye una etapa evolucionaria tardía en la secuencia de desarrollo de esta enfermedad desde una lesión precursora (3). En la mayoría de los cánceres epiteliales, uno de los cambios identificables más precoces en las lesiones precursoras es la activación de la maquinaria reparadora del DNA y la acumulación nuclear de p53 $(32,48)$.

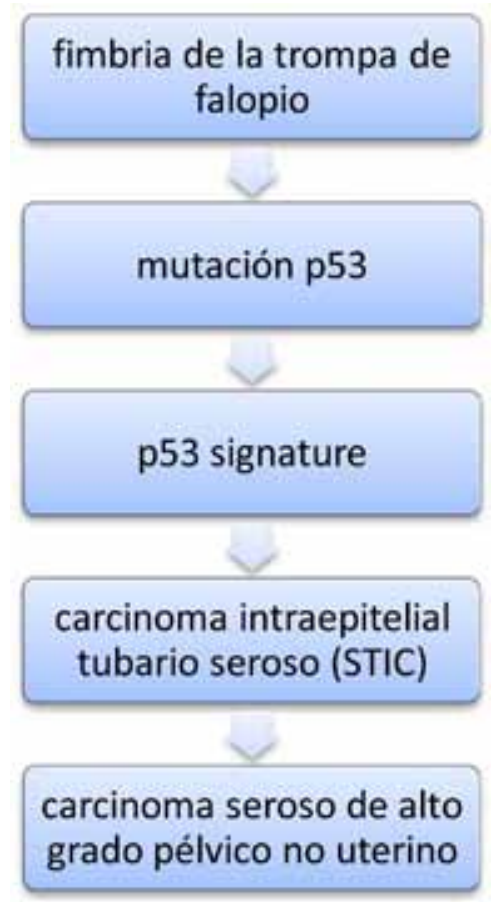

Figura 1. Vía molecular del carcinoma seroso de alto grado del ovario (CSPAG). Adaptado de referencia (53) 


\title{
Tabla I
}

\section{EVIDENCIA DE QUE LA TROMPA DE FALOPIO ES EL ORIGEN DEL CSPAG *}

1. Proximidad de las fimbrias a los ovarios y a las superficies peritoneales

2. Alta frecuencia de CIT en salpingo-ooforectomías profilácticas de mujeres BRCA+

3. Identificación de CIT en alto porcentaje de carcinomas pélvicos serosos consecutivos clasificados como tubario, peritoneal primario y ovárico (31)

4. Status de mutación p53 compartido entre CIT y carcinoma seroso pélvico (31)

* 1 y 2 resumidos también en referencia (24). Modificada de referencia (31).

Tabla II

EVIDENCIAS DE LA ASOCIACION ENTRE UN PRECURSOR (p53 SIGNATURE), STICY CSPAG *

\author{
- Predilección por la fimbria \\ - Aparición en situación de daño de DNA (g-H2AX+) \\ - Célula secretora tubaria: la probable originaria de las 3 entidades \\ - Marcada acumulación nuclear de p53 \\ - Mutaciones p53 asociadas a CSPAG \\ - Continuidad documentada entre p53 signature y STIC \\ - Formas intermedias entre p53 signature y STIC (61)
}

* Modificada de referencia (34)

El examen de los ovarios y trompas de Falopio de 75 mujeres BRCA+, detectó 29 "p53 signatures" en mucosa tubaria, sólo 1 en el epitelio de la superficie ovárica (ESO) y 0 en quistes de inclusión corticales, confirmando que "p53 signatures" se originan preferentemente en el epitelio de la trompa de Falopio (ETF) en vez de en el ESO (49).

Se ha demostrado sobrepoblación de células secretoras en segmentos del epitelio de la trompa de Falopio, entidad denominada SCOUT (sobrecrecimiento de células secretoras en la trompa, por sus siglas en inglés), significativamente más prevalente en trompas de pacientes con cáncer pélvico seroso que en trompas de pacientes portadoras de mutación BRCA, y que en trompas de controles. Esta entidad sería biológicamente análoga a "p53 signature". Presenta expresión alterada de múltiples genes dentro de un epitelio tubario histológicamente benigno, incluyendo el gen PAX2, un gen down regulado en el cáncer seroso de endometrio. La mayoría de los SCOUT exhibe pérdida de PAX2. "p53 signatures", STIC y carcinoma seroso pélvico evidencian presencia conjunta de anomalías en la expresión de p53 y PAX2, hallazgo concordante con la naturaleza multigénica de la carcinogénesis (50). La presencia en SCOUT de anomalías genéticas (PAX2 y p53) tan fuertemente ligadas a cáncer pélvico seroso, refuerza más el concepto de que la trompa de Falopio es un sitio relevante de iniciación de este cáncer (50).

CSETF humanas aisladas de muestras de trompas de Falopio normales sanas, inmortalizadas, y transformadas con oncogenes, originaron carcinomas müllerianos de alto grado macroscópica, histológica, inmunofenotípica, y genómicamente similares al CSPAG humano (51).

Al inducir en ratón doble mutación (gen Dicer esencial para la síntesis de micro RNA -supresores tumorales-, y gen $\mathrm{PTEN}$ regulador negativo clave de la vía de PI3KA) éste desarrolla cáncer intraperitoneal seroso de alto grado con similitud macroscópica, histológica, inmunohistoquímica y genético molecular, al CSPAG humano. Al extirpar tempranamente las trompas de Falopio en forma 
exclusiva (dejando intactos los ovarios), en el ratón no se desarrolló carcinoma seroso de alto grado. Inversamente, al extirparles los ovarios y conservar las trompas, se observó el desarrollo del cáncer, concluyendo que la trompa de Falopio es el sitio donde se inicia y desarrolla el CSPAG (52).

\section{PATOGENIA MOLECULAR DEL CSPAG}

Involucra múltiples cambios genéticos y epigenéticos. En más del $90 \%$ de los casos existe mutación somática en TP53 (19). La pérdida de la función de TP53 debe estar acompañada por lo menos por un evento genotóxico adicional (incluyendo la inactivación funcional de BRCA 1/2) para producir el fenotipo maligno (19).

\section{IMPLICANCIAS CLÍNICAS}

La identificación de la trompa de Falopio como el origen del CSPAG, y de su lesión precursora asociada, tiene un impacto clínico potencial significativo en reducción de su mortalidad (53). El $20 \%$ de las pacientes diagnosticadas con cáncer ovárico ha tenido cirugía ginecológica previa (sin salpingectomía) y $10-15 \%$ ligadura tubaria previa (54). Con salpingectomía en la cirugía inicial, 30\% de los cánceres ováricos se podría haber prevenido. La salpingectomía durante la histerectomía o esterilización tiene mínimo riesgo quirúrgico adicional. Al comparar 79 pacientes en las cuales se realizó histerectomía total laparoscópica (HTL) con salpingectomía bilateral con 79 pacientes en las que se realizó HTL sin salpingectomía, no hubo diferencia significativa en tiempo operatorio, caída en hemoglobina, estadía hospitalaria, retorno a la actividad normal o tasa de complicaciones entre ambos grupos (55). Varias instituciones han recomendado salpingectomía bilateral profiláctica para disminuir la incidencia del CPSAG $(56,57,58,59)$. Se ha demostrado aumento de salpingectomía sin incremento de riesgo operatorio o complicaciones perioperatorias (56).

Al comparar mujeres con cirugía previa por indicación benigna (esterilización, salpingectomía, histerectomía, y salpingo-ooforectomía bilateral; $n=251.465)$, con la población no expuesta $(n=5.449 .119)$, hubo una reducción del riesgo y estadísticamente significativo para cáncer ovárico en las mujeres con salpingectomía previa $(\mathrm{HR}=0,65$; IC95\% 0,52 a 0,81) (60).

\section{CONSIDERACIONES FINALES}

La mayoría de los carcinomas ováricos son del subtipo histológico seroso de alto grado. Este subgrupo difiere marcadamente de los otros subtipos histológicos de cáncer ovárico en morfología, orígenes carcinogénicos, y a nivel molecular. Estas características morfológicas, carcinogénicas y genético-moleculares son a su vez compartidas por los CSPAG, lo que hace apropiado y necesario considerar a éstos como un grupo definido y excluyente de los demás cánceres ováricos. La evidencia acumulada demuestra su origen en la trompa de Falopio, y confiere a la salpingectomía bilateral un probable rol preventivo para esta patología. La importancia clínica de reconocer en la trompa de Falopio el origen del CSPAG está en que esta enfermedad podría ser interrumpida antes de su diseminación a la superficie ovárica y pélvica. Se han presentado los datos que respaldan el concepto de que STIC es un paso establecido en la secuencia carcinogénica serosa.

\section{REFERENCIAS}

1. Bast R, Hennessy B, Mills GB. The biology of ovarian cancer: New opportunities for translation. Nat Rev Cancer 2009,9:415-28.

2. American Cancer Society,2007. Cancer Facts and Figures. www.cancer.org.

3. Ferlay J, Shin B, Bray F, et al. Globocan 2008 v2.0, Cancer Incidence and Mortality Worldwide: IARC Cancer Base No. 10 [internet]. Lyon, France. In: International Agency for Research on Cancer; 2010.

4. Lowe K, Chia V, Taylor A, et al. An international assessment of ovarian cancer incidence and mortality. Gynecol Oncol 2013;130:107-14.

5. Cuello M. GES en cáncer de ovario epitelial: un avance sanitario necesario pero no exento de riesgos y dificultades futuras. Rev Chil Obstet Ginecol 2013;78(3):161-6.

6. Seidman J, Horkayne-Szakaly I, Haiba M, et al. The histologic type and stage distribution of ovarian carcinomas of surface epithelial origin. Int J Gynecol Pathol 2004;23:41-4.

7. Gurung A, Hung T, Morin J, Gilks B. Molecular abnormalities in ovarian carcinoma: clinical, morphological and therapeutic correlates. Histopathology 2013,62,59-70.

8. Vang R, Shih I, Kurman R. Ovarian low-grade and high-grade serous carcinoma: Pathogenesis, clinic pathologic and molecular biologic features, and diagnostic problems. Adv Anat Pathol 2009,16:267-82.

9. Ahyan A, Kurman R, Vang R,et al. Defining the cutpoint between low- and high-grade ovarian serous carcinomas: A clinic pathologic and molecular genetic analysis. Am J Surg Pathol2009;33:1220-4.

10. Malpica A, Deavers MT, Lu K, et al. Grading ovarian serous carcinoma using a two-tier system. Am J Surg Pathol 2004;28:496-504.

11. Kurman R, Shih I. Pathogenesis of ovarian cancer: lessons from morphology and molecular biology and their clinical implications. Int J Gynecol Pathol 2008,27:151-60.

12. Shih I, Kurman R. Ovarian tumorigenesis: a proposed model based on morphological and molecular genetic analysis. Am J Pathol 2004,164:1511-8.

13. Seidman J, Cho K, Ronnett B, Kurman R. Surface epithelial tumors of the ovary. En: Kurman RJ, Ellenson 
$\mathrm{LH}$, Ronnett BM, editors. Blaustein's Pathology of the Female Genital Tract. 6th ed. 2011. New York: Springer.

14. Singer G, Stohr R, Cope L, et al. Patterns of p53 mutations separate ovarian serous borderline tumors and low- and high-grade carcinomas and provide support for a new model of ovarian carcinogenesis: a mutational analysis with immunohistochemical correlation. Am J Surg Pathol 2005;29:218-24.

15. Kuo K, Guan B, Feng Y, et al. Analysis of DNA copy number alterations in ovarian serous tumors identifies new molecular genetic changes in low-grade and high-grade carcinomas. Cancer Res 2009;69:403642.

16. Ahmed A, Etemadmoghadam D, Temple J, et al. Driver mutations in TP53 are ubiquitous in high grade serous carcinoma of the ovary. J Pathol 2010;221:4956.

17. Kurman R, Shih I. The origin and pathogenesis of epithelial ovarian cancer: a proposed unifying theory. Am J Surg Pathol 2010;34:433-43.

18. Landen C, Birrer M, Sood A. Early events in the pathogenesis of epithelial ovarian cancer. J Clin Oncol 2008;26:995-1005.

19. Haruta S, Furukawa N, Yoshizawa Y, et al. Molecular genetics and epidemiology of epithelial ovarian cancer (Review). Oncol Reports 2011;26:1347-56.

20. Brewer M, Johnson K, Follen M, et al. Prevention of ovarian cancer: intraepithelial neoplasia. Clin Cancer Res 2003;9:20-30.

21. Risch $\mathrm{H}$, McLaughlin J, Cole $\mathrm{D}$, et al. Population BRCA1 and BRCA2 mutation frequencies and cancer penetrances: a kin-cohort study in Ontario, Canada.J Natl Cancer Instit 2006;98:1694-706.

22. Wooster R, Weber B. Breast and ovarian cancer.N Engl J Med 2003;348:2339-47.

23. Lancaster J, Powell C, Kauff N, et al. Society of Gynecologic Oncologists Education Committee statement on risk assessment for inherited gynecologic cancer predispositions. Gynecol Oncol 2007;107:159-62.

24. Piek J, van Diest $P$, Zweemer R, et al. Dysplastic changes in prophylactically removed Fallopian tubes of women predisposed to developing ovarian cancer. J Pathol 2001;195:451-6.

25. Medeiros F, Muto M, Lee $Y$, et al. The tubal fimbria is a preferred site for early adenocarcinoma in women with familial ovarian cancer syndrome. Am J Surg Pathol 2006;30:230-6.

26. Leeper K, Garcia R, Swisher E, et al: Pathologic findings in prophylactic oophorectomy specimens in high-risk women. Gynecol Oncol 2002;87:52-6.

27. Powell C, Kenley E, Chen L, et al. Risk-reducing salpingo-oophorectomy in BRCA mutation carriers: role of serial sectioning in the detection of occult malignancy. J Clin Oncol 2005;23:127-32.

28. Finch A, Shaw $P$, Rosen B, et al. Clinical and pathologic findings of prophylactic salpingo-oophorectomies in 159 BRCA1 and BRCA2 carriers. Gynecol Oncol 2006;100:58-64.

29. Callahan M, Crum C, Medeiros F, et al. Primary fallopian tube malignancies in BRCA-positive women undergoing surgery for ovarian cancer risk reduction. $\mathrm{J}$ Clin Oncol 2007;25:3985-90.

30. Crum C, Drapkin R, Kindelbereger D, et al. Lessons from BRCA: the tubal fimbria emerges as an origin for pelvic serous cancer. Clin Med Res 2007;5:35-44.
31. Kindelberger D, Lee Y, Miron A, et al. Intraepithelial carcinoma of the fimbria and pelvic serous carcinoma: Evidence for a causal relationship. Am J Surg Pathol 2007;31:161-9.

32. Levanon K, Crum C, Drapkin R. New insights into the pathogenesis of serous ovarian cancer and its clinical impact. J Clin Oncol 2008;26:5284-93

33. Karst A, Drapkin R. Ovarian cancer pathogenesis: a model in evolution. J Oncol 2010: Article ID 932371.

34. Crum C. Intercepting pelvic cancer in the distal faIlopian tube: Theories and realities. Mol Oncol 2009;3:165-70.

35. Carlson J, Miron A, Jarboe E, et al: Serous tubal intraepithelial carcinoma: its potential role in primary peritoneal serous carcinoma and serous cancer prevention. J Clin Oncol 2008;26:4160-5.

36. Roh M, Kindelberger D, Crum C. Serous tubal intraepithelial carcinoma and the dominant ovarian mass clues to serous tumor origin? Am J Surg Pathol 2009;33:376-83.

37. Nonaka D, Chiriboga L, Soslow R. Expression of PAX8 as a useful marker in distinguishing ovarian carcinomas from mammary carcinomas. Am J Surg Pathol 2008;32:1566-71.

38. Bowen N, Logani S, Dickerson E, et al. Emerging roles for PAX8 in ovarian cancer and endosalpingeal development. Gynecol Oncol 2007;104:331-7.

39. Lee $\mathrm{Y}$, Miron A, Drapkin R, et al. A candidate precursor to serous carcinoma that originates in the distal fallopian tube. J Pathol 2007;211:26-35.

40. Singer G, Stohr R, Cope L, et al. Patterns of p53 mutations separate ovarian serous borderline tumors and low- and high-grade carcinomas and provide support for a new model of ovarian carcinogenesis: a mutational analysis with immunohistochemical correlation. Am J Surg Pathol 2005;29:218-24.

41. Chiesa-Vottero A, Malpica A, Deavers M, et al. Immunohisto- chemical over expression of p16 and p53 in uterine serous carcinoma and ovarian high-grade serous carcinoma. Int J Gynecol Pathol 2007;26:32833.

42. O'Neill C, McBride H, Connolly L, et al. High-grade ovarian serous carcinoma exhibits significantly higher p16 expression than low-grade serous carcinoma and serous borderline tumour. Histopathology 2007;50:773-9.

43. Rogakou E, Boon C, Redon C, Bonner W. Megabase chromatin domains involved in DNA double- strand breaks in vivo. J Cell Biol1999;146:905-15.

44. Bartkova J, Horejsi Z, Koed K, et al. DNA damage response as a candidate anti-cancer barrier in early human tumorigenesis. Nature 2005;434:864-70.

45. Gorgoulis V, Vassiliou L, Karakaidos P, et al. Activation of the DNA damage checkpoint and genomic instability in human precancerous lesions. Nature 2005;434:907-13.

46. Levine A. p53, the cellular gatekeeper for growth and division. Cell 1997;88:323-31.

47. Zhang Z, Fu G, Wang M, et al. P53 codon 72 polymorphism and ovarian cancer risk: a meta-analysis. J Nanjing Med University 2008;22:279-85.

48. Halazonetis T, Gorgoulis V, Bartek J. An oncogeneinduced DNA damage model for cancer development. Science 2008;319:1352-5.

49. Folkins $A$, Jarboe $E$, Callahan $M$, et al. A candidate precursor to pelvic serous cancer (p53 Signature) 
and its prevalence in ovaries and fallopian tubes from women with heterozygous BRCA mutations. Gynecol Oncol 2008;109:168-73.

50. Chen E, Mehra K, Mehrad M, et al. Secretory cell outgrowth, PAX2 and serous carcinogenesis in the Fallopian tube. J Pathol 2010;222:110-6.

51. Karst A, Levanon K, Drapkin R. Modeling high-grade serous ovarian carcinogenesis from the fallopian tube. Proc Natl Acad Sci USA 2011;108:7547-52.

52. Kim J, Coffey D, Creighton C, et al. High-grade serous ovarian cancer arises from fallopian tube in a mouse model. Proc Natl Acad Sci USA 2012;109:3921-6.

53. RCOG. The distal fallopian tube as the origin of nonuterine pelvic high-grade serous carcinomas. Scientific Impact Paper No 44. Nov 2014. (c) Royal College of Obstetricians and Gynecologists.

54. Tone A, Salvador S, Finlayson S, et al. The role of the fallopian tube in ovarian cancer. Clin Adv Hematol Oncol 2012;10:296-306.

55. Morelli M, Venturella R, Mocciaro R, et al. Prophylactic salpingectomy in premenopausal low-risk women for ovarian cancer: primum non nocere. Gynecol Oncol 2013;129:448-51.
56. McAlpine J, Hanley G, Woo M, et al. Ovarian cancer research program of British Columbia. Opportunistic salpingectomy: uptake, risks, and complications of a regional initiative for ovarian cancer prevention. Am J Obstet Gynecol 2014;210:471.e1-e11.

57. Royal Australian and New Zealand College of Obstetricians and Gynaecologists. Managing the adnexae at the time of hysterectomy for benign gynaecological disease. College Statement C-Gyn 25. Melbourne, Australia: RANZCOG; 2012.

58. Society of Gynecologic Oncology. SGO Clinical Practice Statement: Salpingectomy for ovarian cancer prevention. Chicago: SGO; 2013. [https://www.sgo. org/clinical- practice/guidelines/sgo-clinical-practicestatement-salpingectomy-for-ovarian-cancer- prevention].

59. ACOG Committee Opinion. Number 620. Jan 2015. Salpingectomy for Ovarian Cancer Prevention.

60. Falconer H, Yin L, Grönberg H, Altman D. Ovarian cancer risk after salpingectomy: a nationwide population-based study. J Natl Cancer Inst 2015;107(2). pii:dju410.

61. Jarboe E, Folkins A, Nucci MR, et al. Serous carcinogenesis in the fallopian tube: a descriptive classification. Int J Gynecol Pathol 2008;27:1-9. 